\title{
A Framework for Analyzing Probabilistic Protocols and its Application to the Partial Secrets Exchange
}

\author{
Konstantinos Chatzikokolakis and Catuscia Palamidessi \\ LIX, École Polytechnique, 91128 Palaiseau, France
}

\begin{abstract}
We propose a probabilistic variant of the pi-calculus as a framework to specify randomized security protocols and their intended properties. In order to express an verify the correctness of the protocols, we develop a probabilistic version of the testing semantics. We then illustrate these concepts on an extended example: the Partial Secret Exchange, a protocol which uses a randomized primitive, the Oblivious Transfer, to achieve fairness of information exchange between two parties.
\end{abstract}

\section{Introduction}

Probabilistic security protocols involve probabilistic choices and are used for many purposes including signing contracts, sending certified email and protecting the anonymity of communication agents. Some probabilistic protocols rely on specific random primitives such as the Oblivious Transfer ([12]). There are various examples in this category, notably the contract signing protocol in [4] and the privacy-preserving auction protocol in [7].

A large effort has been dedicated to the formal verification of security protocols, and several approaches based on process-calculi techniques have been proposed. However, in the particular case of probabilistic protocols, they have been analyzes mainly by using model checking methods, while only few attempts of applying process calculi techniques have been made. The only work we are aware of is [2], which defines a probabilistic version of the noninterference property, and uses a probabilistic variant of CCS and of bisimulation to analyse protocols wrt this property.

In this paper we present a framework for analyzing probabilistic security protocols using the $\pi_{\text {prob }}$-calculus, a probabilistic extension of the $\pi$-calculus inspired by the work in [5]. In order to express security properties in this calculus, we extend the notion of testing equivalence ([8]) to the probabilistic setting. We propose a preorder based on the probability of passing a certain class of tests: a process $P$ is considered smaller than a process $Q$ if, for each test, the probability of passing it is smaller for $P$ than for $Q$. Following the lines of [1], a test can be seen as an adversary who interacts with an agent in order to break some security property. In order to verify a security property, then, we can create a specification which satisfies it and show that the protocol is smaller than 
the specification with respect to the testing preorder. If this holds, then the adversary has smaller probability of succeeding with the protocol than with the specification, so the protocol is correct with respect to the examined property.

We illustrate the above described framework with an extended example of fair exchange protocol, where the property to verify is fairness. In this type of protocols two agents, $A$ and $B$, want to exchange information simultaneously, that is each of them is willing to send its secrets only if he receives the ones of the other party. We consider the Partial Secrets Exchange protocol (PSE, [4]) which uses the Oblivious Transfer as its main primitive. An important characteristic of fair exchange protocols is that the adversary is in fact one of the agents and not an external party. As a consequence the behavior of $A$ will be different when $B$ behaves normally than when he is trying to cheat. After encoding the protocol in the $\pi_{p r o b}$-calculus, we give a specification which models the behavior of $A$ in case he is being cheated. We then express fairness by means of a testing relation between the protocol and the specification and we prove that it holds.

The rest of the paper is organized as follows: in the next section we introduce $\pi_{\text {prob }}$, our variant of the probabilistic $\pi$-calculus. We present its semantics and propose a notion of probabilistic testing preorder. In Section 3 we illustrate the Oblivious Transfer primitive, the Partial Secrets Exchange protocol (PSE), and their encoding in the $\pi_{\text {prob }}$-calculus. In Section 4 we specify the fairness property and we prove the correctness of PSE. In Section 5 we discuss related work, notably the analysis of the PSE protocol using probabilistic model checking. Finally, Section 6 concludes and presents some ideas for future work.

For reasons of space, the proofs have been removed from the main text. They can be found in the Appendix.

\section{A probabilistic variant of $\pi$-calculus}

In this section we define a probabilistic process calculus suitable for implementing security protocols. This calculus, which will be referred as the $\pi_{p r o b}$-calculus, is a probabilistic extension of the $\pi$-calculus, similar to the probabilistic asynchronous $\pi$-calculus presented in [5].

A common feature of $\pi_{p r o b}$ and the calculus in [5] is that there is a distinction between probabilistic and deterministic behavior. The first, represented by the choice operator, is associated with the random choices performed by the process itself. The second, represented by the parallel operator, is related to the decisions of an external scheduler.

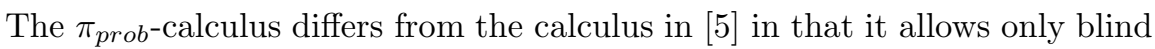
choices. This simplifies considerably semantics and reasoning, while the calculus remains rich enough to model probabilistic security protocols. Furthermore, the $\pi_{p r o b}$-calculus contains some extra constructs, like output prefix and pair splitting, that are useful to express the protocols we have considered.

We could also add certain cryptographic primitives like the shared-key encryption of the spi-calculus, however this wasn't necessary in the protocols examined so far. 


\subsection{Syntax}

Let $x, y$ range over a countable set of variables and $n, m$ over a countable set of process names. The terms and processes of the $\pi_{p r o b}$-calculus are defined by the following grammar:

\begin{tabular}{|c|c|c|c|}
\hline$T::=$ & term & $P::=$ & process \\
\hline$x$ & variable & $\bar{T} T . P$ & output \\
\hline | $n$ & name & $T(x) . P$ & input \\
\hline $\mid\langle T, T\rangle$ & pair & $|P| P$ & composition \\
\hline & & $\sum_{i} p_{i} P_{i}$ & prob. choice \\
\hline & & $\nu n P$ & restriction \\
\hline & & $! P$ & replication \\
\hline & & | $[T$ is $T] P$ & match \\
\hline & & $\begin{array}{l}\mid \text { let }\langle x, y\rangle=T \text { in } P \\
0\end{array}$ & $\begin{array}{l}\text { pair splitting } \\
\text { nil }\end{array}$ \\
\hline
\end{tabular}

The distinction between variables and channel names does not exist in the original $\pi$-calculus but simplifies the treatment of some relations.

\subsection{Probabilistic automata}

The semantics of $\pi_{\text {prob }}$ is based on Probabilistic Automata, which were introduced in [13]. We briefly recall here the main notions, simplified and adapted for our needs.

A discrete probabilistic space is a pair $(X, p b)$ where $X$ is a set and $p b$ a function $p b: X \mapsto(0,1]$ s.t. $\sum_{x \in X} p b(x)=1$. Given a set $Y$ we define the set of all probabilistic spaces on $Y$ :

$\operatorname{Prob}(Y)=\{(X, p b) \mid X \subseteq Y$ and $(X, p b)$ is a discrete probabilistic space $\}$

Let $S$ be a set of states and $A$ a set of actions. A probabilistic automaton is a triple $\left(\mathcal{S}, \mathcal{T}, s_{0}\right)$ where $s_{0} \in S$ (initial state) and $\mathcal{T} \subseteq S \times \operatorname{Prob}(A \times S)$. The elements of $\mathcal{T}$ are called transition groups. The idea is that the choice between transition groups is made non-deterministically by an external scheduler while the choice of a transition within a group is made probabilistically by the process itself.

Given a probabilistic automaton $M=\left(\mathcal{S}, \mathcal{T}, s_{0}\right)$ we define $\operatorname{tree}(M)$ as the tree obtained by unfolding the transition system. The root $n_{0}$ of $\operatorname{tree}(M)$ is labeled by $s_{0}$ and if $n$ is a node labeled by $s$ then for each $(s,(X, p b)) \in \mathcal{T}$ and each $\left(\mu, s^{\prime}\right) \in X$ there is a node $n^{\prime}$ labeled by $s^{\prime}$ and an arc from $n$ to $n^{\prime}$ labeled by $\mu$ and $p b\left(\mu, s^{\prime}\right)$.

A scheduler $\zeta$ is a function which solves the nondeterminism by selecting, at each moment of the computation, a transition group among the ones allowed at the current state. The execution tree of an automaton $M$ under a scheduler $\zeta$, denoted by etree $(M, \zeta)$ is the tree obtained from tree $(M)$ by pruning all the arcs corresponding to transitions in groups not selected by $\zeta$. 


$$
\begin{aligned}
& \text { IN } \quad m(x) . P\{\underset{1}{\stackrel{m(x)}{\longrightarrow}} P\} \quad \text { OUT } \quad \bar{m} M . P\{\underset{1}{\stackrel{\bar{m} M}{\longrightarrow}} P\} \\
& \mathrm{SUM} \quad \sum_{i} p_{i} P_{i}\left\{\underset{p_{i}}{\stackrel{\tau}{\longrightarrow}} P_{i}\right\}_{i} \\
& \operatorname{OPEN} \frac{P\left\{\underset{1}{\stackrel{\bar{m} n}{\longrightarrow}} P^{\prime}\right\}}{\nu n P\left\{\stackrel{\bar{m}(n)}{\longrightarrow} \nu n P^{\prime}\right\}} \quad m \neq n \\
& \text { RES1 } \frac{P\left\{\frac{\mu}{1} P^{\prime}\right\}}{\nu n P\left\{\frac{\mu}{1} \nu n P^{\prime}\right\}} \quad \begin{array}{c}
\mu \neq \tau, \\
n \notin f n(\mu)
\end{array} \\
& \text { RES2 } \frac{P\left\{\frac{\tau}{p_{i}} P_{i}\right\}_{i}}{\nu n P\left\{\underset{p_{i}}{\stackrel{\tau}{\longrightarrow}} \nu n P_{i}\right\}_{i}} \\
& \mathrm{COM} \frac{P\left\{\underset{1}{\stackrel{\bar{m} M}{\longrightarrow}} P^{\prime}\right\} \quad Q\left\{\stackrel{\frac{m(x)}{1}}{\longrightarrow} Q^{\prime}\right\}}{P \mid Q\left\{\stackrel{\tau}{\longrightarrow} P^{\prime} \mid Q^{\prime}[M / x]\right\}} \\
& \operatorname{PAR} \frac{P\left\{\frac{\mu_{i}}{p_{i}} P_{i}\right\}_{i}}{P \mid Q\left\{\frac{\mu_{i}}{p_{i}} P_{i} \mid Q\right\}_{i}} \quad \forall i f n\left(\mu_{i}\right) \cap b n(Q)=\emptyset \\
& \operatorname{CLOSE} \frac{P\left\{\stackrel{\bar{m}(n)}{\longrightarrow} P^{\prime}\right\} \quad Q\left\{\frac{m(x)}{1} Q^{\prime}\right\}}{P \mid Q\left\{\underset{1}{\longrightarrow} \nu n\left(P^{\prime} \mid Q^{\prime}[n / x]\right)\right\}} \\
& \mathrm{CONG} \frac{P \equiv P^{\prime} \quad P^{\prime}\left\{\frac{\mu_{i}}{p_{i}} Q_{i}^{\prime}\right\}_{i} \quad \forall i \cdot Q_{i}^{\prime} \equiv Q_{i}}{P\left\{\frac{\mu_{i}}{p_{i}} Q_{i}\right\}_{i}}
\end{aligned}
$$

Fig. 1. The late-instantiation semantics of the $\pi_{p r o b}$-calculus. The functions $f n$ and $b n$ give respectively the free and the bound names of the argument.

\subsection{Semantics of $\pi_{p r o b}$}

The operational semantics of the $\pi_{\text {prob }}$-calculus is given by means of probabilistic automata defined inductively on the basis of the syntax. In order to simplify the notation, we write

$$
s\left\{\underset{p_{i}}{\stackrel{\mu_{i}}{\longrightarrow}} \mid i \in I\right\}
$$

iff $\left(s,\left(\left\{\left(\mu_{i}, s_{i}\right) \mid i \in I\right\}, p b\right)\right) \in \mathcal{T}$ and $\forall i \in I: p_{i}=p b\left(\mu_{i}, s_{i}\right)$, where $I$ is an index set. When $I$ is not relevant we will use the notation $s\left\{\frac{\mu_{i}}{p_{i}}\right\}_{i}$.

The transitions of the automaton associated to a process are defined by the rules in Figure 1.

The behavior of the choice operator is defined by the SUM rule. The transition to every member of the sum is possible with a $\tau$ action (blind choice). Note that all transitions belong to the same group which means that the choice is not controlled by the scheduler but is made by the process itself. IN and OUT are self-explanatory. RES models restriction on channel $n$ : actions on that channel are not allowed by the restricted process. Note that we have split this rule in two for the sake of clarity: for the transition groups which contain only $\tau$ actions there is no need to check the channel name. PAR models interleaving, in which each process maintains its transition groups. COM models communication by handshaking. Since input/output transitions are always alone in their group, this rule is rather simple and very similar to the non-probabilistic case. CLOSE is similar to COM but works together with OPEN in order to implement scope 
extrusion, that is the transfer of a new channel name between processes. Finally CONG states that equivalent processes perform the same actions. The structural equivalence $\equiv$ used in CONG is defined as follows:

$$
\begin{aligned}
& \text { ( } \alpha \text {-renaming) } P \equiv Q \quad \text { iff } P \equiv_{\alpha} Q \\
& P \mid 0 \equiv P \\
& P|Q \equiv Q| P \\
& ! P \equiv P \mid ! P \\
& \text { let }\langle x, y\rangle=\langle M, N\rangle \text { in } P \equiv P[M / x][N / y] \quad[M \text { is } M] P \equiv P
\end{aligned}
$$

In the following sections we define some relations between $\pi_{\text {prob }}$ processes which will help us expressing some properties of probabilistic protocols and reasoning about them. We will also examine some properties of these relations.

\subsection{Testing relations between $\pi_{p r o b}$ processes}

Testing is a well-known method of comparing processes, resulting in equivalences weaker than the ones of the bisimulation family. The idea, proposed by De Nicola and Hennessy ([8]), is that two processes are equivalent if they both pass the same set of tests. A test is a process running in parallel with the one being tested and which can perform a distinguished action $\omega$ which represents success. This idea is very useful for the analysis of security protocols, as suggested in [1], since a test can be seen as an adversary who interferes with a communication agent and declares his success with an $\omega$ action. Then two processes are testing equivalent if they are vulnerable to the same attacks.

In the probabilistic setting there are different approaches for defining testing equivalence. For example [11] proposes a probabilistic extension of testing equivalence which considers the ability of each process to pass a test with nonzero probability (may testing) or probability one (must testing). However, when analyzing security protocols we are not only interested in the ability of passing a test, but also in the exact probability of success. Thus our definition resembles more to the one of [6] and the result is no longer an equivalence but a preorder.

We start by defining the probability of a set of executions. Given a probabilistic automaton $M$ and a scheduler $\zeta$, an execution fragment $\xi$ is a path (finite or infinite) from the root of etree $(M, \zeta)$. The probability of an execution fragment $\xi=n_{0} \underset{p_{0}}{\stackrel{\mu_{0}}{\longrightarrow}} n_{1} \underset{p_{1}}{\stackrel{\mu_{1}}{\longrightarrow}} n_{2} \underset{p_{2}}{\stackrel{\mu_{2}}{\longrightarrow}} \ldots$ is defined as $p b(\xi)=\prod_{i} p_{i}$. An execution is a maximal execution fragment. The set of all executions of $M$ under $\zeta$ is denoted by $\operatorname{exec}(M, \zeta)$.

Given an execution fragment $\xi$, a cone with prefix $\xi$ is defined as $C_{\xi}=\left\{\xi^{\prime} \in\right.$ $\left.\operatorname{exec}(M, \zeta) \mid \xi \leq \xi^{\prime}\right\}$ where $\leq$ is the prefix relation. We define $p b\left(C_{\xi}\right)=p b(\xi)$. Let $\left\{C_{i}\right\}_{i \in I}$ be a countable set of disjoint cones. We define $p b\left(\bigcup_{i \in I} C_{i}\right)=$ $\sum_{i \in I} p b\left(C_{i}\right)$. We can show that this probability is well defined, that is two different sets of disjoint cones with the same union give the same probability.

A test $O$ is a $\pi_{p r o b}$-calculus process able to perform a distinguished action $\omega$. An interaction between $O$ and a process $P$ is a sequence of $\tau$ transitions starting from $P \mid O$. In order to allow only $\tau$ actions we define $\nu P=\nu n_{1} \ldots \nu_{n} P$, where $\nu_{1}, \ldots, \nu_{n}$ are all the free names in $P$. Then an interaction between $P$ and $O$ is 
an element of $\operatorname{exec}(\nu(P \mid O), \zeta)^{1}$ :

$$
\nu(P \mid O)=Q_{0} \underset{p_{0}}{\stackrel{\tau}{\longrightarrow}} Q_{1} \underset{p_{1}}{\stackrel{\tau}{\longrightarrow}} Q_{2} \underset{p_{2}}{\stackrel{\tau}{\longrightarrow}} \ldots
$$

An interaction $\xi$ is successful if $Q_{i} \underset{p}{\stackrel{\omega}{p}}$ for some $i$. Let $\operatorname{sexec}(\nu(P \mid O), \zeta)=\{\xi \in$ $\operatorname{exec}(\nu(P \mid O), \zeta) \mid \xi$ is successful $\}$. This set can be obtained as a countable union of disjoint cones [5], so the probability of a successful execution can be defined as $p b(\operatorname{sexec}(\nu(P \mid O), \zeta))$.

We now define the upper and lower probability for $P$ to pass $O$.

Definition 1. Let $P$ be a process and $O$ a test. We define

$$
\begin{aligned}
& P\lceil O\rceil=\sup \{p b(\operatorname{sexec}(\nu(P \mid O), \zeta)) \mid \zeta \text { is a scheduler }\} \\
& P\lfloor O\rfloor=\inf \{p b(\operatorname{sexec}(\nu(P \mid O), \zeta)) \mid \zeta \text { is a scheduler }\}
\end{aligned}
$$

Then we define the testing preorders for $\pi_{\text {prob }}$-processes.

Definition 2. Let $P, Q$ be processes. We define must and may-testing preorders as follows:

$$
\begin{aligned}
& P \sqsubseteq \text { may } Q \quad \text { iff for all tests } O: P\lceil O\rceil \leq Q\lceil O\rceil \\
& P \sqsubseteq{ }_{\text {must }} Q \quad \text { iff for all tests } O: P\lfloor O\rfloor \leq Q\lfloor O\rfloor
\end{aligned}
$$

In this paper we will only use may-testing to express safety properties of security protocols, so we will write just $\sqsubseteq$ for $\sqsubseteq$ may

As we will see in the following sections, an agent of a probabilistic security protocol behaves differently when his partner deviates from the protocol in an attempt to cheat. In order to model this behavior we introduce a conditional testing preorder, which is exactly the same as may-testing except that it only considers tests who satisfy a certain condition.

Definition 3. Let $P, Q$ be processes. We define the conditional may-testing preorder as follows:

$$
P \sqsubseteq^{\phi} Q \quad \text { iff for all tests } O: \phi(O) \Rightarrow P\lceil O\rceil \leq Q\lceil O\rceil
$$

where $\phi(O)$ is a condition on $O$.

Finally we define a useful preorder between pairs of processes:

Definition 4. Let $P_{1}, P_{2}, Q_{1}, Q_{2}$ be processes. We define the relation $\sqsubseteq_{p}$ between pairs of processes as follows

$$
\left(P_{1}, P_{2}\right) \sqsubseteq_{p}\left(Q_{1}, Q_{2}\right) \quad \text { iff } \quad P_{1}+{ }_{p} P_{2} \sqsubseteq Q_{1}+{ }_{p} Q_{2}
$$

where $P_{1}+{ }_{p} P_{2}$ stands for $\sum_{i=1}^{2} p_{i} P_{i}$ with $p_{1}=p$ and $p_{2}=1-p$.

\footnotetext{
${ }^{1}$ With a slight abuse of notation we will sometimes use a process to denote its corresponding probabilistic automaton.
} 


\subsection{Properties of testing preorders}

In this section we will examine some properties of the previously defined relations. We present only the corresponding lemmas, all proofs can be found in the appendix.

The following lemma is very useful for reasoning about the upper probability of passing a test. It crucially relies on the fact that in $\pi_{\text {prob }}$ probabilistic choices are blind.

Lemma 1. Let $P, Q$ be $\pi_{\text {prob }}$ processes and $p \in[0,1]$. Then for all tests $O$

$$
P+{ }_{p} Q\lceil O\rceil=p P\lceil O\rceil+(1-p) Q\lceil O\rceil
$$

A context $C$ is a process containing a "hole". We will denote by $C[P]$ the process obtained by replacing the hole in $C$ by $P$. A preorder is a precongruence if it is closed under any context.

May-testing is not a precongruence on arbitrary processes since for $P=$ $[x$ is $y] P^{\prime}, Q=[x$ is $z] Q^{\prime}, C=n(x)$.[ ], we have $P \sqsubseteq Q$ but $C[P] \sqsubseteq C[Q]$ does not hold for all $P^{\prime}, Q^{\prime}$. However all previous relations become precongruences if we consider only closed processes, that is those without free variables.

Lemma 2. $\sqsubseteq_{p}$ is a precongruence on closed processes, that is for all contexts $C$ and all closed processes $P_{1}, P_{2}, Q_{1}, Q_{2}$

$$
\left(P_{1}, P_{2}\right) \sqsubseteq_{p}\left(Q_{1}, Q_{2}\right) \Rightarrow\left(C\left[P_{1}\right], C\left[P_{2}\right]\right) \sqsubseteq_{p}\left(C\left[Q_{1}\right], C\left[Q_{2}\right]\right)
$$

The following corollary is an immediate consequence of lemmas 1 and 2 .

Corollary 1. $\sqsubseteq$ is a precongruence on closed processes.

\section{Probabilistic Security Protocols}

In this section we discuss probabilistic security protocols based on the Oblivious Transfer and we show how to model them using the $\pi_{\text {prob-calculus. }}$

\subsection{1-out-of-2 Oblivious Transfer}

The Oblivious Transfer is a primitive operation used in various probabilistic security protocols. In this particular version a sender $A$ sends exactly one of the messages $M_{1}, M_{2}$ to a receiver $B$. The latter receives $i$ and $M_{i}$ where $i$ is 1 or 2, each with probability $1 / 2$. Moreover $A$ should get no information about which message was received by $B$. More precisely the protocol $\mathrm{OT}_{2}^{1}\left(A, B, M_{1}, M_{2}\right)$ should satisfy the following conditions:

1. If $A$ executes $\mathrm{OT}_{2}^{1}\left(A, B, M_{1}, M_{2}\right)$ properly then $B$ receives exactly one message, $\left(1, M_{1}\right)$ or $\left(2, M_{2}\right)$, each with probability $1 / 2$.

2. After the execution of $\mathrm{OT}_{2}^{1}\left(A, B, M_{1}, M_{2}\right)$, if it is properly executed, for $A$ the probability that $B$ got $M_{i}$ remains $1 / 2$. 
3. If $A$ deviates from the protocol, in order to increase his probability of learning what $B$ received, then $B$ can detect his attempt with probability at least $1 / 2$.

It is worth noting that in the literature the reception of the index $i$ by $B$ is often not mentioned, at least not explicitly ([4]). However, omitting the index can lead to possible attacks. Consider the case where $A$ executes (properly) $\mathrm{OT}_{2}^{1}\left(M_{1}, M_{1}\right)$. Then $B$ will receive $M_{1}$ with probability one, but he cannot distinguish it from the case where he receives $M_{1}$ as a result of $\mathrm{OT}_{2}^{1}\left(M_{1}, M_{2}\right)$. So $A$ is forcing $B$ to receive $M_{1}$. We will see that, in the case of the PSE protocol, $A$ could exploit this situation in order to get an unfair advantage. Note that the condition 3 does not apply to this situation since this cannot be considered as a deviation from the Oblivious Transfer. A generic implementation of the Oblivious Transfer could not detect such behavior since $A$ executes OT properly, the problem lies only in the data being transfered.

Using the indexes, however, solves the problem since $B$ will receive $\left(2, M_{1}\right)$ with probability one half. This is distinguishable from any outcome of $\mathrm{OT}_{2}^{1}\left(M_{1}\right.$, $M_{1}$ ) so, in the case of PSE, $B$ could detect that he's being cheated. Implementations of the Oblivious Transfer do provide the index information, even though sometimes it is not mentioned ([4]). In other formulations of the OT the receiver can actually select which message he wants to receive, so this problem is irrelevant.

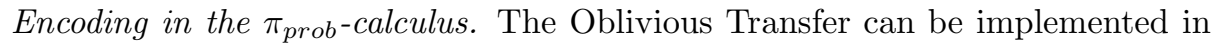

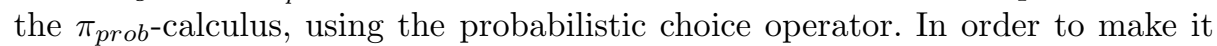
impossible to cheat, a server process is used to coordinate the transfer. The processes of the sender and the server are the following:

$$
\begin{aligned}
\mathrm{OT}_{2}^{1}\left(m_{1}, m_{2}, c_{a s}\right) & \triangleq \overline{c_{a s}} m_{1} \cdot \overline{c_{a s}} m_{2} \cdot 0 \\
S\left(c_{a s}, c_{s b}\right) & \triangleq c_{a s}\left(m_{1}\right) \cdot c_{a s}\left(m_{2}\right) \cdot\left(\overline{c_{b s}}\left\langle 1, m_{1}\right\rangle+0.5 \overline{c_{b s}}\left\langle 2, m_{2}\right\rangle\right)
\end{aligned}
$$

where $m_{1}, m_{2}$ are the names to be sent. $c_{a s}$ is a channel private to $A$ and $S$ and $c_{s b}$ a channel private to $B$ and $S$. Each agent communicates only with the server and not directly with the other agent. $B$ receives the message from the server (who should be in parallel with $A$ and $B$ ) by making an input action on $c_{s b}$.

It is easy to see that these processes correctly implement the Oblivious Transfer. The only requirement is that $A$ should not contain $c_{s b}$, so that he can only communicate with $B$ through the server.

\subsection{Partial Secrets Exchange Protocol}

This protocol is the core of three probabilistic protocols for contract signing, certified email and coin tossing, all presented in [4]. It involves two agents, each having $2 n$ secrets split in pairs, $\left(a_{1}, a_{n+1}\right), \ldots,\left(a_{n}, a_{2 n}\right)$ for $A$ and $\left(b_{1}, b_{n+1}\right), \ldots$, $\left(b_{n}, b_{2 n}\right)$ for $B$. Each secret consists of $m$ bits. The purpose is to exchange a single pair of secrets under the constraint that, if at a specific time $B$ has one 


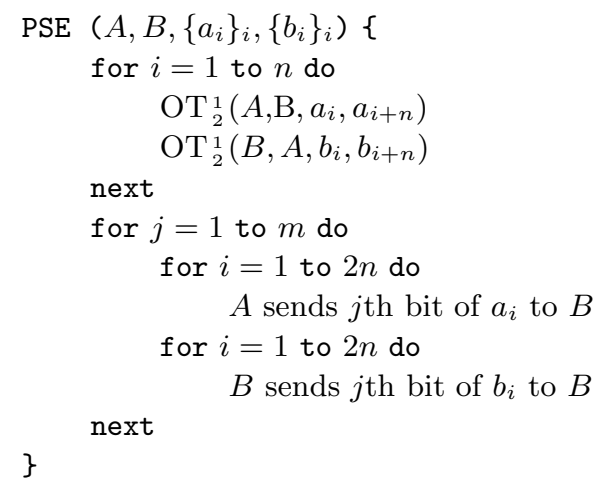

Fig. 2. Partial Secrets Exchange protocol

of $A$ 's pairs, then with high probability $A$ should also have one of $B$ 's pairs and vice versa.

The protocol, displayed in figure 2, consists of two parts. During the first $A$ and $B$ exchange their pairs of secrets using $\mathrm{OT}_{2}^{1}$. After this step $A$ knows exactly one half of each of $B$ 's pairs and vice versa. During the second part, all secrets are exchanged bit per bit. Half of the bits received are already known from the first step, so both agents can check whether they are valid. Obviously, if both $A$ and $B$ execute the protocol properly then all secrets are revealed.

The problem arises when $B$ tries to cheat and sends incorrectly some of his secrets. In this case it can be proved that with high probability some of the tests of $A$ will fail causing $A$ to stop the execution of the protocol and avoid revealing his secrets. The idea is that, in order for $B$ to cheat, he must send at least one half of each of his pairs incorrectly. However he cannot know which of the two halves is already received by $A$ during the first part of the protocol. So a pair sent incorrectly will have only one half probability of being accepted by $A$, leading to a total $2^{-n}$ probability of success.

Now imagine, as discussed in section 3.1 , that $B$ executes $\mathrm{OT}_{2}^{1}\left(B, A, b_{i}, b_{i}\right)$, thus forcing $A$ to receive $b_{i}$. Now, in the second part, he can send all $\left\{b_{i+n} \mid 1 \leq\right.$ $i \leq n\}$ incorrectly without failing any test. Moreover $A$ cannot detect this situation. If indexes are available $A$ will receive $\left(2, b_{i+n}\right)$ with probability one half and since he knows that $b_{i+n}$ is not the second half of the corresponding pair (secrets are recognizable) he will stop the protocol.

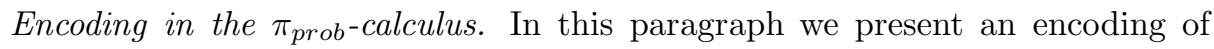
the PSE protocol in the $\pi_{\text {prob-calculus. Before giving the corresponding process }}$ there are two points worth discussing.

- The secrets exchanged by PSE should be recognizable, which means that agent $A$ cannot compute $B$ 's secrets, but he can recognize them when he receives them. Of course a secret can be recognized only as a whole, no single bit can be recognized by itself. To implement this feature we allow $B$ 's secrets 


$$
\begin{aligned}
& A\left(\left\{a_{i j}\right\}_{i=1 . .2 n, j=1 . . m},\left\{b_{i}\right\}_{i=1 . .2 n}\right) \triangleq \\
& \prod_{i=1}^{n} \mathrm{OT}_{2}^{1}\left(\left\langle a_{i 1}, \ldots, a_{i j}\right\rangle,\left\langle a_{(i+n) 1}, \ldots, a_{(i+n) j}\right\rangle, c_{a s_{i}}\right) \mid \\
& c_{s a_{1}}\left(\left\langle k_{1}, r_{1}\right\rangle\right) . \text { let }\left\langle r_{11}, \ldots, r_{1 j}\right\rangle=r_{1} \text { in } \ldots c_{s a_{n}}\left(\left\langle k_{n}, r_{n}\right\rangle\right) \text {.let }\left\langle r_{n 1}, \ldots, r_{n j}\right\rangle=r_{n} \text { in } \\
& \nu q_{1} \ldots \nu q_{n+1}\left(\overline{q_{1}} \star\left|\prod_{i=1}^{n} q_{i}(x) \cdot \operatorname{TestOT}(i)\right|\right. \\
& \quad q_{n+1}(x) . \nu s_{1} \ldots \nu s_{m+1}\left(\overline{s_{1}} \star \mid\right. \\
& \quad \prod_{j=1}^{m} s_{j}(x) \cdot \overline{c_{p}} a_{1 j} \ldots \overline{c_{p}} a_{(2 n) j} \cdot c_{p}\left(d_{1 j}\right) \ldots c_{p}\left(d_{(2 n) j}\right) . \\
& \nu t_{1} \ldots \nu t_{n+1}\left(\overline{t_{1}} \star\left|\prod_{i=1}^{n} t_{i}(x) \cdot \operatorname{Test}(i, j)\right| t_{n+1}(x) \cdot \overline{s_{j+1}} \star\right) \mid \\
& \left.\left.\quad s_{m+1}(x) \cdot \overline{c_{p}} o k\right)\right)
\end{aligned}
$$

$\operatorname{TestOT}(i) \triangleq\left(\left[k_{i}\right.\right.$ is 1$]\left[r_{i}\right.$ is $\left.b_{i}\right] \overline{q_{i+1}} \star \mid\left[k_{i}\right.$ is 2$]\left[r_{i}\right.$ is $\left.\left.b_{i+n}\right] \overline{q_{i+1}} \star\right)$

$\operatorname{Test}(i, j) \triangleq\left(\left[k_{i}\right.\right.$ is 1$]\left[r_{i j}\right.$ is $\left.d_{i j}\right] \overline{t_{i+1}} \star \mid\left[k_{i}\right.$ is 2$]\left[r_{i j}\right.$ is $\left.\left.d_{(i+n) j}\right] \overline{t_{i+1}} \star\right)$

Fig. 3. Encoding of PSE protocol

to appear in $A$ 's process, as if $A$ knew them. However we allow a secret to appear only as a whole (not decomposed) and only inside a test construct, which means that it can only be used to recognize another message.

- In our analysis we need to detect the fact that an agent sends a specific bit of a specific message. If all bits are the same then this is difficult. Thus, in the implementation of PSE, each parameter $a_{i j}$ (resp. $b_{i j}$ ) can be considered to take values from the domain $\left\{0_{i j}, 1_{i j}\right\}$, where $0_{i j}$ (resp. $1_{i j}$ ) is a public channel but different for each $i, j$.

Note that having secrets composed by public bits can lead to guessing attacks by non-deterministic adversaries. Many analysis tools for security protocols, such as the spi-calculus, do not allow the decomposition of secrets to avoid such guesses. In our analysis, however, we express the correctness of a protocol as the equivalence with a properly constructed specification. This only proves that the protocol will not reveal any secrets and is not related with the adversary's ability of guessing the secrets without interfering with any partner (of course, this is known to happen with very small probability). Such attacks will apply to both the protocol and the specification.

The encoding for the general case of $n$ pairs and $m$ bits per message is displayed in figure 3 . We denote by $a_{i}$ (resp. $\left.b_{i}\right)$ the $i$-th secret of $A$ (resp. $B$ ) and by $a_{i j}$ (resp. $b_{i j}$ ) the $j$-th bit of $a_{i}$ (resp. $b_{i}$ ). $r_{i}$ is the $i$-th message received by Oblivious Transfer and $k_{i}$ is the corresponding index.

The first part consists of the first 3 lines of the process definition. In this part $A$ sends his pairs using $\mathrm{OT}_{2}^{1}$, receives the ones of $B$ and decomposes them. To check the received messages $A$ starts a loop of $n$ steps, each of whom is guarded by an input action on $q_{i}$ for synchronization. During the $i$-th step, $r_{i}$ is tested against $b_{i}$ or $b_{i+n}$ depending on the outcome of the OT, that is on the value of $k_{i}$. 
The second part consists of a loop of $m$ steps, each of whom is guarded by an input action on $s_{j}$. During each step the $j$-th bit of each secret is sent and the corresponding bits of $B$ are received in $d_{i j}$. Then there is nested loop of $n$ tests controlled by the input actions on $t_{i}$. Each test, performed by the Test subprocess, ensures that $B$ 's bits are valid. Test $(i, j)$ checks the $j$-th bit of the $i$-th pair. The bit received during the first part, namely $r_{i j}$, is compared to $d_{i j}$ or $d_{(i+n) j}$ depending on $k_{i}$. If the bit is valid, an output action on $t_{i+j}$ is performed to continue to the next test.

Finally, an instance of the protocol is an agent $A$ put in parallel with servers for all oblivious transfers:

$$
I \triangleq A\left(\left\{a_{i j}\right\}_{i=1 . .2 n, j=1 . . m},\left\{b_{i}\right\}_{i=1 . .2 n}\right) \mid \prod_{i=1}^{n}\left(S\left(c_{a s_{i}}, c_{s b_{i}}\right) \mid S\left(c_{b s_{i}}, c_{s a_{i}}\right)\right)
$$

\section{Verification of Security Properties}

A well known method for expressing and proving security properties using process calculi is by means of specifications. A specification $P_{\text {spec }}$ of a protocol $P$ is a process which is simple enough in order to prove (or accept) that it models the correct behavior of the protocol. Then the correctness of $P$ is implied by $P \simeq P_{\text {spec }}$ where $\simeq$ is a testing equivalence. The idea is that, if there exist an attack for $P$, this attack can be modeled by a test $O$ which performs the attack and outputs $\omega$ if it succeeds. Then $P$ should pass the test and since $P \simeq P_{\text {spec }}$, $P_{\text {spec }}$ should also pass it, which is a contradiction (no attack exists for $P_{\text {spec }}$ ).

However, in case of probabilistic protocols, attacks do exist but only succeed with a very small probability. So examining only the ability of passing a test is not sufficient since the fact that $P_{\text {spec }}$ has an attack is no longer contradictory. Instead we will use a specification which can be shown to have very small probability of been attacked and we will express the correctness of $P$ as $P \sqsubseteq P_{\text {spec }}$ where $\sqsubseteq$ is the testing preorder defined in section 2.4. Then an attack of high probability for $P$ should be applicable with at least the same probability for $P_{\text {spec }}$ which is contradictory.

\subsection{A specification for PSE}

Let us recall the fairness property for the PSE protocol.

If $B$ receives one of $A$ 's pairs then with high probability $A$ should also be able to receive one of $B$ 's pairs.

First of all we must point out two important differences between this type of protocols and the traditional cryptographic ones.

- In traditional protocols both $A$ and $B$ are considered honest. The purpose of the protocol is to ensure that no outside adversary can access the messages being transfered. 


$$
\begin{aligned}
& A_{\text {spec }}\left(\left\{a_{i j}\right\}_{i=1 . .2 n, j=1 . . m}, h\right) \triangleq \\
& \prod_{i=1}^{n} \mathrm{OT}_{2}^{1}\left(\left\langle a_{i 1}, \ldots, a_{i j}\right\rangle,\left\langle a_{(i+n) 1}, \ldots, a_{(i+n) j}\right\rangle, c_{a s_{i}}\right) \mid \\
& c_{s a_{1}}(x) \ldots c_{s a_{n}}(x) \text {. } \\
& \nu q_{1} \ldots \nu q_{n+1}\left(\overline{q_{1}} \star \mid \prod_{i=1}^{n} q_{i}(x) \cdot \text { TestOT }_{\text {spec }}(i) \mid\right. \\
& q_{n+1}(x) . \nu s_{1} \ldots \nu s_{m+1}\left(\overline{s_{1}} \star \mid\right. \\
& \prod_{j=1}^{m} s_{j}(x) \cdot \overline{c_{p}} a_{1 j} \ldots \overline{c_{p}} a_{(2 n) j} \cdot c_{p}(x) \ldots c_{p}(x) . \\
& \nu t_{1} \ldots \nu t_{n+1}\left(\overline{t_{1}} \star\left|\prod_{i=1}^{n} t_{i}(x) \cdot \operatorname{Test}_{\text {spec }}(i, j, h)\right| t_{n+1}(x) . \overline{s_{j+1}} \star\right) \mid \\
& \left.\left.s_{m+1}(x) \cdot \overline{c_{p}} o k\right)\right) \\
& \text { Test } O T_{\text {spec }}(i) \triangleq \overline{q_{i+1}} \star
\end{aligned}
$$

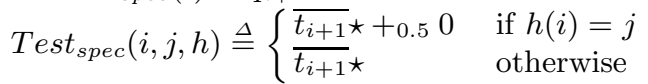

Fig. 4. Specification for the PSE protocol

On the other hand, in PSE the adversary is $B$ himself, who might try to deviate from the protocol in order to get $A$ 's secrets without revealing his own ones.

- In traditional protocols the secrets must remain secret all the time. $A$ and $B$ always perform the same actions and always want to communicate with each other.

On the other hand in PSE $A$ shows different behavior when $B$ is honest than in case of an attempt to cheat. $A$ is willing to reveal his secrets, only when $B$ wants the same too.

A specification of a protocol shows the correct behavior of the agents. Since $A$ 's behavior depends on $B$ it makes sense to have different specifications depending on $B$ 's behavior. Since the case where $B$ is honest is trivial, we are considering the case where $B$ tries to deviate from the protocol. That is $B$ will try to send some of his bits incorrectly. Moreover the behavior of $A$ depends on which these bits are. If $B$ stays honest for the first half bits then $A$ will do the same.

It order to model $B$ 's intention to cheat, we will use a function $h:\{1 . . n\} \mapsto$ $\{1 . . m\}$ who shows on which bit $B$ is going to cheat for each pair. So $h(3)=4$ means that $B$ is going to send the 4 th bit of (at least) one of the 3rd pair's secrets incorrectly. Note that, in order for $B$ to cheat, $h$ must be defined on its whole domain (otherwise at least one pair will be sent correctly). However, concerning the specification, $h$ could be a partial function.

The specification is displayed in figure 4 . As already discussed, it depends on $B$ 's cheating behavior, that is on the function $h$. The specification resembles a lot the protocol, with two major differences: 
1. The specification does not use any of its input (all input variables are replaced by $x$ to point out this fact). Moreover $b_{i}$ 's are no longer used (thus they removed from the parameter list).

2. The specification does not test the received bits. In the first part, TestOT $T_{\text {spec }}$ accepts all messages. In the second, Test ${ }_{\text {spec }}$ accepts all bits, except those on which $B$ is known to cheat, which are accepted only with probability one half.

As a consequence the specification is much simpler than the protocol. As we will show in the next section, if $h$ is total then $A$ will make $n$ choices and the probability of succeeding in all of them will be negligible.

\subsection{Proving the correctness of PSE}

Correctness of the specification. First we show that the specification is indeed a proper specification for PSE with respect to fairness, in case $B$ tries to cheat. Let $l$ be the maximum number of bits that $B$ is willing to reveal for its secrets. So $B$ 's cheating behavior will be described by a function $h$, such that $h(i) \leq l+1$ for all $i \in\{1 . . n\}$. This is by definition of PSE, otherwise $B$ would reveal $l+1$ bits of at least one pair of secrets and one pair is enough for $A$.

As we already discussed $A_{\text {spec }}$ does not depend on its input. Moreover it is deterministic, that is only one transition is possible at any moment, except from Test $_{\text {spec }}(i, j, h)$ for $h(i)=j$ where the process stalls with probability one half. Since $h(i) \leq l+1, \forall i \in\{1 . . n\}$, all $n$ of these tests will appear in the first $l+1$ steps of the second part of the protocol. If $A$ fails in even one test then he stalls, so the total probability of advancing to step $l+2$ and reveal its $l+2$ pair is $2^{-n}$.

This means that $A_{\text {spec }}$ satisfies fairness. If $B$ at some point of the protocol has $l$ bits of one of $A$ 's pairs, then with probability at least $1-2^{-n} A$ will have $l-1$ bits of at least one of $B$ 's pairs. If $l=m$ ( $B$ has a whole pair) then $A$ should have at least $m-1$ bits and the last bit can be easily computed by trying both 0 and 1 . In other words $B$ cannot gain an advantage of more than one bit with probability greater than $2^{-n}$.

Relation between $A$ and $A_{\text {spec }}$. Having proved the correctness of the specification with respect to fairness, it rests to show its relation with the original protocol. Proving $A \sqsubseteq A_{\text {spec }}$ means to prove that if $A$ is vulnerable with high probability to an attack $O$, then $A_{\text {spec }}$ will be also vulnerable with at least the same probability. Since we know that the probability of a successful attack for $A_{\text {spec }}$ is very small, we can conclude that an attack on $A$ is very unlikely.

Note however that $A_{\text {spec }}$ models the behavior of $A$ only in case of an attack described by the function $h$. So $A \sqsubseteq A_{\text {spec }}$ cannot hold in general since $A$ will pass with greater probability a test who models a honest agent. Thus, we need to use the conditional may-testing defined in section 2.4. 
An instance of the specification is a process $A_{\text {spec }}$ put in parallel with servers for all oblivious transfers:

$$
I_{s p e c}(h) \triangleq A\left(\left\{a_{i j}\right\}_{i=1 . .2 n, j=1 . . m}, h\right) \mid \prod_{i=1}^{n}\left(S\left(c_{a s_{i}}, c_{s b_{i}}\right) \mid S\left(c_{b s_{i}}, c_{s a_{i}}\right)\right)
$$

Let $H$ be the set of all total functions $h:\{1 . . n\} \mapsto\{1 . . m\}$. PSE will be considered correct wrt fairness if:

$$
\begin{aligned}
& \forall h \in H: I \sqsubseteq^{\phi_{h}} I_{\text {spec }}(h) \\
& \text { where } \phi_{h}(O)=\text { true iff } \forall i \in\{1 . . n\}: \\
& \qquad O \text { does not contain both } b_{i h(i)} \text { and } b_{(i+n) h(i)}
\end{aligned}
$$

The condition $\phi_{h}$ ensures that the test will try to cheat on the $h(i)$-th bit of each pair $i$. The idea is that in order to cheat, an intruder should refuse to send at least one bit of each message.

We can now state the correctness of PSE, as defined above.

Theorem 1. PSE is correct with respect to fairness.

\section{Related Work}

Security protocols have been extensively studied during the last decade and many formal methods have been proposed for their analysis. However, the vast majority of these methods refer to non-deterministic protocols and are not suitable for the probabilistic setting, since they do not allow to model random choices. One exception is the work of Aldini and Gorrieri ([2]), where they use a probabilistic process algebra to analyze fairness in a non-reputation protocol. Their work is close to ours in spirit, although technically it is quite different. In particular, we base our analysis on a notion of testing while theirs is based on a notion of bisimulation.

With respect to the application, the results the most related to ours come from Norman and Schmatikov ([9], [10]), who use probabilistic model checking to study fairness in two probabilistic protocols, including the Partial Exchange Protocol. In particular, in [10] they model the PSE using Prism, a probabilistic model checker. Their treatment however is very different from ours: their model describes only the "correct" behavior for both $A$ and $B$, as specified by the protocol. $B$ 's ability to cheat is limited to prematurely stopping the execution, so attacks in which $B$ deviates completely from the protocol are not taken into account. Having a simplified model is important in model checking since it helps overcoming the search state explosion problem, thus making the verification feasible.

The results in [10] show that with probability one $B$ can gain a one bit advantage, that is he can get all $m$ bits of a pair of $A$ by revealing only $m-1$ bits of his. This is achieved simply by stopping the execution after receiving the last bit from $A$. Moreover a method of overcoming the problem is proposed, which 
gives this advantage to $A$ or $B$, each with probability one half. Is is worth noting that this is a very weak form of attack and could be considered as negligible, since $A$ can compute the last bit very easily by trying both 0 and 1 . Besides a one bit advantage will always exist in contract signing protocols, simply because synchronous communication is not feasible.

In our approach, by modeling an adversary as an arbitrary $\pi_{\text {prob }}$ process we allow him to perform a vast range of attacks including sending messages, performing calculations, monitoring public channels etc. Our analysis shows not only that a one bit attack is possible, but more important that no attack to obtain an advantage of two or more bits exists with non-negligible probability. Moreover our method has has the advantage of being easily extendable. For example, treating more sessions, even an infinite number of ones, can be done by putting many copies of the processes in parallel.

Of course, the major advantage of the model checking approach, with respect to ours, is that it can be totally automated.

\section{Conclusion}

In this paper we examined a method to analyze probabilistic security protocols using process calculi. The main tool for this analysis is the $\pi_{\text {prob-calculus, a prob- }}$ abilistic variant of the $\pi$-calculus. The probabilistic choice, provided by $\pi_{\text {prob }}$, allowed us to encode the Partial Exchange Protocol, a probabilistic protocol based on the Oblivious Transfer. In order to prove the correctness of this protocol, we defined various preorders between $\pi_{\text {prob }}$ processes and examined their properties. Then we presented a properly constructed specification and showed that it is stronger that the original protocol, thus proving that the possibility of success for any attack is very small.

Our results show that process calculi techniques can be successfully applied to security protocol analysis. There are various advantages of this approach. First of all the use of process calculi allows the use of various tools from the corresponding theory. The proofs obtained are general, covering every possible adversary and are not instance-based as in model checking techniques. Moreover process calculi allow the analysis of a protocol in a more complex environment, having for example many agents and multiple simultaneous instances of a protocol. It is worth noting that many attacks of well known protocols only appear in such situations.

In [3] an algorithm for deciding may-testing is presented, for fully probabilistic automata. We believe that this result can be extended to the probabilistic automata defined in section 1.2, giving the ability of automatically proving the correctness of probabilistic security protocols.

\section{References}

1. Martn Abadi and Andrew Gordon. A calculus for cryptographic protocols: The spi calculus. Information and Computation, 148(1):1-70, 1999. 
2. Alessandro Aldini and Roberto Gorrieri. Security analysis of a probabilistic nonrepudiation protocol. In Holger Hermanns and Roberto Segala, editors, Proceedings of the Second Joint International Workshop PAPM-PROBMIV 2002, volume 2399 of Lecture Notes in Computer Science, page 17, Heidelberg, 2002. Springer-Verlag.

3. L. Christoff and I. Christoff. Efficient algorithms for verification of equivalences for probabilistic processes. In Larsen and Skou, editors, Proc. Workshop on Computer Aided Verification, volume 575 of LNCS. Springer Verlag, 1991.

4. Shimon Even, Oded Goldreich, and Abraham Lempel. A randomized protocol for signing contracts. Commun. ACM, 28(6):637-647, 1985.

5. Oltea Mihaela Herescu and Catuscia Palamidessi. Probabilistic asynchronous $\pi$ calculus. In Jerzy Tiuryn, editor, Proceedings of FOSSACS 2000 (Part of ETAPS 2000), Lecture Notes in Computer Science, pages 146-160. Springer-Verlag, 2000.

6. Bengt Jonsson, Kim G. Larsen, and Wang Yi. Probabilistic extensions of process algebras. Handbook of Process Algebras, 2001.

7. Moni Naor, Benny Pinkas, and Reuban Sumner. Privacy preserving auctions and mechanism design. In Proceedings of the 1st ACM conference on Electronic commerce, pages 129-139. ACM Press, 1999.

8. R. De Nicola and M. C. B. Hennessy. Testing equivalences for processes. Theoretical Computer Science, 34:83-133, 1984.

9. Gethin Norman and Vitaly Shmatikov. Analysis of probabilistic contract signing. In A. Abdallah, P. Ryan, and S. Schneider, editors, Proc. BCS-FACS Formal Aspects of Security (FASec'02), volume 2629 of LNCS, pages 81-96. Springer, 2003.

10. Gethin Norman and Vitaly Shmatikov. Analysis of probabilistic contract signing. Formal Aspects of Computing (to appear), 2005.

11. Catuscia Palamidessi and Oltea M. Herescu. A randomized encoding of the picalculus with mixed choice. In Proceedings of the 2nd IFIP International Conference on Theoretical Computer Science, pages 537-549, 2002. A full version of this paper has been accepted for publication on Theoretical Computer Science.

12. M Rabin. How to exchange secrets by oblivious transfer. Technical Memo TR-81, Aiken Computation Laboratory, Harvard University, 1981.

13. Roberto Segala and Nancy Lynch. Probabilistic simulations for probabilistic processes. Nordic Journal of Computing, 2(2):250-273, Summer 1995. 


\section{A Proofs}

We present here the proofs that were omitted from the paper.

Lemma 1. Let $P, Q$ be processes and $p \in[0,1]$. For all tests $O$

$$
P+{ }_{p} Q\lceil O\rceil=p P\lceil O\rceil+(1-p) Q\lceil O\rceil
$$

Proof We will write $P(O, \zeta)$ for $\operatorname{pr}(\operatorname{sexec}(\nu(P \mid O), \zeta))$. Firstly we prove that

$$
\exists \zeta: P+{ }_{p} Q(O, \zeta)=\pi \Leftrightarrow \exists \zeta_{1}, \zeta_{2}: p P\left(O, \zeta_{1}\right)+(1-p) Q\left(O, \zeta_{2}\right)=\pi
$$

$\Rightarrow)$ we can construct a scheduler $\zeta^{\prime}$ who performs the choice first and then imitates $\zeta$. It is easy to see that $P+{ }_{p} Q(O, \zeta)=P+{ }_{p} Q\left(O, \zeta^{\prime}\right)$. Moreover all executions of $P+{ }_{p} Q \mid O$ under $\zeta^{\prime}$ will start with one of the following transitions:

$$
P+{ }_{p} Q|O \underset{1}{\stackrel{\tau}{\longrightarrow}} P| O \quad P+{ }_{p} Q|O \underset{1-p}{\stackrel{\tau}{\longrightarrow}} Q| O
$$

and continue with an execution of $P \mid O$ or $Q \mid O$. Thus $P\left(O, \zeta^{\prime}\right)=p P\left(O, \zeta_{1}\right)+$ $(1-p) Q\left(O, \zeta_{2}\right)=\pi$, where $\zeta_{1}, \zeta_{2}$ are schedulers who imitate $\zeta^{\prime}$ after the choice. $\Leftarrow)$ we use for $\zeta$ a scheduler who first selects to do the choice $P+{ }_{p} Q$ and then imitates $\zeta_{1}$ or $\zeta_{2}$ depending on the outcome of the choice.

Now let $A=p P\lceil O\rceil+(1-p) Q\lceil O\rceil$ and suppose that $A \neq \sup \{P+p$ $Q(O, \zeta) \mid \zeta\}$. Then, by definition of sup, one of the following must hold.

1. $\exists \zeta: P+{ }_{p} Q(O, \zeta)>A$. Then by $(1) \exists \zeta_{1}, \zeta_{2}: p P\left(O, \zeta_{1}\right)+(1-p) Q\left(O, \zeta_{2}\right)>A$, so $P\left(O, \zeta_{1}\right)>P\lceil O\rceil$ or $Q\left(O, \zeta_{2}\right)>Q\lceil O\rceil$ which is a contradiction.

2. $\exists A^{\prime}<A$ s.t. $\forall \zeta: P(O, \zeta) \leq A^{\prime}$. Let $\epsilon<A-A^{\prime}$. Since $P\lceil O\rceil, Q\lceil O\rceil$ are the sup of the corresponding sets, there exist $\zeta_{1}, \zeta_{2}$ s.t. $p P\left(O, \zeta_{1}\right)+(1-p) Q\left(O, \zeta_{2}\right)>$ $A-e$. By (1) $\exists \zeta: P+{ }_{p} Q(O, \zeta)>A-e>A^{\prime}$ which is a also contradiction.

To prove lemma 2 we define the open extension of a relation.

Definition 5. Let $\mathcal{R}$ be a relation on closed processes. We define its open extension $R^{\circ}$ on arbitrary processes as $P \mathcal{R}^{\circ} Q$ iff $P \sigma \mathcal{R} Q \sigma$ for all substitutions $\sigma$ such that $P \sigma, Q \sigma$ are closed.

We firstly prove congruence for the open extension of $\sqsubseteq_{p}$.

Lemma 3. $\complement_{p}^{\circ}$ is a precongruence, that is for all contexts $C$ and all processes $P_{1}, P_{2}, Q_{1}, Q_{2}$

$$
\left(P_{1}, P_{2}\right) \sqsubseteq_{p}^{\circ}\left(Q_{1}, Q_{2}\right) \Rightarrow\left(C\left[P_{1}\right], C\left[P_{2}\right]\right) \sqsubseteq_{p}^{\circ}\left(C\left[Q_{1}\right], C\left[Q_{2}\right]\right)
$$


Proof Let $P_{1}, P_{2}, Q_{1}, Q_{2}$ be processes such that $\left(P_{1}, P_{2}\right) \sqsubseteq_{p}^{\circ}\left(Q_{1}, Q_{2}\right)$. By the definition of $\sqsubseteq_{p}^{\circ}$ and by lemma 1 we have that for all tests $O$ and all substitutions $\sigma$ :

$$
p P_{1} \sigma\lceil O\rceil+(1-p) P_{2} \sigma\lceil O\rceil \leq p Q_{1} \sigma\lceil O\rceil+(1-p) Q_{2} \sigma\lceil O\rceil
$$

The proof is done by induction on the structure of $C$. The base case $(C=[])$ is trivial. For the inductive step, we can apply the induction hypothesis to each sub-context, so we have only to examine the following cases.

1. $C=[] \mid R$. The process $R \sigma \mid O$ is by itself a test and it is easy to see that $\forall X: X \mid R \sigma\lceil O\rceil=X\lceil R \sigma \mid O\rceil$. So we have:

$$
\begin{aligned}
& \left(C\left[P_{1}\right] \sigma+_{p} C\left[P_{2}\right] \sigma\right)\lceil O\rceil \\
& =p\left(P_{1} \sigma \mid R \sigma\right)\lceil O\rceil+(1-p)\left(P_{2} \sigma \mid R \sigma\right)\lceil O\rceil \text { lemma } 1 \\
& =p P_{1} \sigma\lceil R \sigma \mid O\rceil+(1-p) P_{2} \sigma\lceil R \sigma \mid O\rceil \\
& \leq p Q_{1} \sigma\lceil R \sigma \mid O\rceil+(1-p) Q_{2} \sigma\lceil R \sigma \mid O\rceil \\
& =p\left(Q_{1} \sigma \mid R \sigma\right)\lceil O\rceil+(1-p)\left(Q_{2} \sigma \mid R \sigma\right)\lceil O\rceil \\
& =\left(C\left[Q_{1}\right] \sigma+{ }_{p} C\left[Q_{2}\right] \sigma\right)\lceil O\rceil
\end{aligned}
$$

thus $\left(C\left[P_{1}\right], C\left[P_{2}\right]\right) \sqsubseteq_{p}^{\circ}\left(C\left[Q_{1}\right], C\left[Q_{2}\right]\right)$.

2. $C=[]+{ }_{k} R$. We have

$$
\begin{aligned}
& \left(C\left[P_{1}\right] \sigma+{ }_{p} C\left[P_{2}\right] \sigma\right)\lceil O\rceil \\
& =p\left(P_{1} \sigma+_{k} R \sigma\right)\lceil O\rceil+(1-p)\left(P_{2} \sigma+{ }_{k} R \sigma\right)\lceil O\rceil \quad \text { lemma } 1 \\
& =p\left(k P_{1} \sigma\lceil O\rceil+(1-k) R \sigma\lceil O\rceil\right)+ \\
& (1-p)\left(k P_{2} \sigma\lceil O\rceil+(1-k) R \sigma\lceil O\rceil\right) \quad \text { lemma } 1 \\
& =k\left(p P_{1} \sigma\lceil O\rceil+(1-p) P_{2} \sigma\lceil O\rceil\right)+(1-k) R \sigma\lceil O\rceil \\
& \leq k\left(p Q_{1} \sigma\lceil O\rceil+(1-p) Q_{2} \sigma\lceil O\rceil\right)+(1-k) R \sigma\lceil O\rceil \\
& =p\left(Q_{1} \sigma+_{k} R \sigma\right)\lceil O\rceil+(1-p)\left(Q_{2} \sigma+_{k} R \sigma\right)\lceil O\rceil \quad \text { lemma } 1 \\
& =\left(C\left[Q_{1}\right] \sigma+{ }_{p} C\left[Q_{2}\right] \sigma\right)\lceil O\rceil \quad \text { lemma } 1
\end{aligned}
$$

thus $\left(C\left[P_{1}\right], C\left[P_{2}\right]\right) \sqsubseteq_{p}^{\circ}\left(C\left[Q_{1}\right], C\left[Q_{2}\right]\right)$.

3. $C=M(x)$.[]. By applying a substitution $\sigma$ to $C[X]$ we get $M \sigma(x) \cdot X \sigma^{\prime}$ where $\sigma^{\prime}$ is the identity substitution for $x$ and equal to $\sigma$ for all other variables.

Without loss of generality we are considering tests who are not performing any actions by themselves before interacting with the tested process. So all applications of a test $O$ to $C[X] \sigma$ will start with the following transition:

$$
\nu\left(M \sigma(x) \cdot X \sigma^{\prime} \mid O\right) \underset{1}{\stackrel{\tau}{\longrightarrow}} \nu\left(X \sigma^{\prime}[x / N] \mid O^{\prime}\right)
$$

Since the probability of this transition is 1 we have

$$
\left(M \sigma(x) . X \sigma^{\prime}\right)\lceil O\rceil=X \sigma^{\prime}[x / N]\left\lceil O^{\prime}\right\rceil
$$


Finally (note that $\sigma^{\prime}[x / N]$ is a substitution)

$$
\begin{aligned}
& \left(C\left[P_{1}\right] \sigma+{ }_{p} C\left[P_{2}\right] \sigma\right)\lceil O\rceil \\
& \quad=p\left(M \sigma(x) \cdot P_{1} \sigma^{\prime}\right)\lceil O\rceil+(1-p)\left(M \sigma(x) \cdot P_{2} \sigma^{\prime}\right)\lceil O\rceil \\
& \left.\quad=p P_{1} \sigma^{\prime}[x / N]\left\lceil O^{\prime}\right\rceil\right)+(1-p) P_{2} \sigma^{\prime}[x / N]\left\lceil O^{\prime}\right\rceil
\end{aligned}
$$

thus $\left(C\left[P_{1}\right], C\left[P_{2}\right]\right) \sqsubseteq_{p}^{\circ}\left(C\left[Q_{1}\right], C\left[Q_{2}\right]\right)$.

4. $C=\bar{M} N$.[]. Similar to the previous case.

5. $C=$ ![]. First we prove that

$$
\left(P_{1}^{n}, P_{2}^{n}\right) \sqsubseteq_{p}^{\circ}\left(Q_{1}^{n}, Q_{2}^{n}\right), \forall n \geq 1
$$

where $P^{n}=P|\ldots| P$ ( $n$ times $)$. The proof is by induction on $n$ and the inductive case is similar to case 1 above (considering $P_{i} \mid O$ and $P_{i}^{n} \mid O$ as tests).

Now suppose that $! P_{1} \sigma+{ }_{p} ! P_{2} \sigma\lceil O\rceil>! Q_{1} \sigma+_{p} ! Q_{2} \sigma\lceil O\rceil$. By choosing a sufficiently large $n$ we can approximate $! P_{i}, ! Q_{i}$ with $P^{n}, Q^{n}$ without invalidating the above inequality, which is contradictory to (4).

6. $C=[M$ is $N][]$. We have $C[X] \sigma=[M \sigma$ is $N \sigma] X \sigma$.

If $M \sigma=N \sigma$ then

$$
[M \sigma \text { is } N \sigma] X \sigma \equiv X \sigma
$$

Thus

$$
\begin{aligned}
& \left(C\left[P_{1}\right] \sigma+{ }_{p} C\left[P_{2}\right] \sigma\right)\lceil O\rceil \\
& \quad=p\left([M \sigma \text { is } N \sigma] P_{1} \sigma\right)\lceil O\rceil+p\left([M \sigma \text { is } N \sigma] P_{2} \sigma\right)\lceil O\rceil \text { lemma } 1 \\
& \quad=p P_{1} \sigma\lceil O\rceil+(1-p) P_{2} \sigma\lceil O\rceil \\
& \quad \leq p Q_{1} \sigma\lceil O\rceil+(1-p) Q_{2} \sigma\lceil O\rceil \\
& \quad=p\left([M \sigma \text { is } N \sigma] Q_{1} \sigma\right)\lceil O\rceil+p\left([M \sigma \text { is } N \sigma] Q_{2} \sigma\right)\lceil O\rceil \\
& \quad=\left(C\left[Q_{1}\right] \sigma+_{p} C\left[Q_{2}\right] \sigma\right)\lceil O\rceil
\end{aligned}
$$

If $M \sigma \neq N \sigma$ then $[M \sigma$ is $N \sigma] X \sigma \equiv 0$ so

$$
C\left[P_{1}\right] \sigma+{ }_{p} C\left[P_{2}\right] \sigma \equiv C\left[Q_{1}\right] \sigma+_{p} C\left[Q_{2}\right] \sigma
$$

and the relation still holds.

7. $C=$ let $\langle x, y\rangle=M$ in []. Similar to the previous case.

Corollary 2. $\sqsubseteq_{p}$ is a precongruence on closed processes. 
Proof We showed that $\sqsubseteq_{p}^{\circ}$ is a precongruence, and for any relation $\mathcal{R}$ if $\mathcal{R}^{\circ}$ is a precongruence on open processes then $\mathcal{R}$ is a precongruence on closed processes.

Corollary $\mathbf{1 .} \sqsubseteq$ is a precongruence on closed processes.

Proof Let $P, Q, R$ be processes such that $P \sqsubseteq Q, C$ be a context and $p \in(0,1)$. For all tests $O, P\lceil O\rceil \leq Q\lceil O\rceil$ so by lemma $1, P+{ }_{p} R \sqsubseteq Q+{ }_{p} R$. By corollary 2 $(C[P], C[R]) \sqsubseteq_{p}(C[Q], C[R])$ and again by lemma $1 C[P]\lceil O\rceil \leq C[Q]\lceil O\rceil$, thus $C[P] \sqsubseteq C[Q]$.

Theorem 1. PSE is correct with respect to fairness.

Proof First we prove that under some conditions $\sqsubseteq^{\phi_{h}}$ is a precongrence. More specifically for all closed processes $P, Q$, if $P \sqsubseteq{ }^{\phi_{h}} Q$ then $C[P] \sqsubseteq{ }^{\phi_{h}} C[Q]$ for all contexts $C$ that do not contain none of $b_{i h_{i}}, \bar{b}_{(i+n) h(i)}$. The proof is similar to the one of lemma 2. The condition above is used in case 1 to ensure that $R \mid O$ satisfies $\phi_{h}$ (and similarly in cases 3 and 4 ).

Now let $h \in H$. We want to prove that $I \sqsubseteq^{\phi_{h}} I_{\text {spec }}$. The two processes differ only in TestOT and Test sub-processes. We define $I_{w}$ to be the same as $I$ after replacing TestOT with TestOT $T_{w}$ and Test with Test Tefined as: $_{w}$

$$
\begin{gathered}
\operatorname{TestOT}_{w}(i) \triangleq \begin{cases}\operatorname{TestOT}(i) & \text { if } i \geq w \\
\operatorname{TestOT} T_{\text {spec }}(i) & \text { otherwise }\end{cases} \\
\text { Test }_{w}(i, j, h) \triangleq \begin{cases}\operatorname{Test}(i, j) & \text { if } i \geq w \\
\operatorname{Test}_{\text {spec }}(i, j, h) & \text { otherwise }\end{cases}
\end{gathered}
$$

The idea is that $I_{w}$ behaves as the specification for the first $w-1$ pairs and as the original protocol for the other ones. Since $I=I_{1}$ and $I_{\text {spec }}=I_{n+1}$ we can prove the correctness of PSE by induction on $w$ and it suffices to show that

$$
I_{w} \sqsubseteq^{\phi_{h}} I_{w+1} \quad \forall w \in\{1 . . n\}
$$

Let $O$ be a test such that $\phi_{h}(O)=$ true. During the execution of $I_{w} \mid O$ variables bounded by input actions in $I_{w}$ will be instantiated to channel names. Let $\xi$ be an execution of $I_{w} \mid O$ and $x^{\prime}$ the name to which variable $x$ is instantiated during this execution. Also let $I_{w}^{\prime}$ be the same as $I_{w}$ after replacing every bound variable $x$ by $x^{\prime}$. Then $I_{w}^{\prime} \mid O$ will be able to perform $\xi$. If we show that for all possible values of $x^{\prime}, I_{w}^{\prime} \sqsubseteq^{\phi_{h}} I_{w+1}^{\prime}$ then $I_{w} \sqsubseteq^{\phi_{h}} I_{w+1}$.

$I_{w}^{\prime}$ and $I_{w+1}^{\prime}$ differ only in the $\operatorname{TestOT}_{w}^{\prime}(i)$ and $\operatorname{Test}_{w}^{\prime}(i, j, h)$ subprocesses for $i=w$. For $j \neq h(w)$ we have:

$$
\begin{aligned}
\operatorname{Test}_{w}^{\prime}(w, j, h)= & {\left[k_{w}^{\prime} \text { is } 1\right]\left[r_{w j}^{\prime} \text { is } d_{w j}^{\prime}\right] \overline{t_{w+1}} \star \mid } \\
& {\left[k_{w}^{\prime} \text { is } 2\right]\left[r_{w j}^{\prime} \text { is } d_{(w+n) j}^{\prime}\right] \overline{t_{w+1}} \star } \\
\operatorname{Test}_{w+1}^{\prime}(w, j, h)= & \overline{t_{w+1} \star}
\end{aligned}
$$


Since $k_{w}^{\prime}$ can only have one value, the one branch of $\operatorname{Test}_{w}^{\prime}(w, j, h)$ will stall. If any of the tests of the other branch fails then $\operatorname{Test}_{w}^{\prime}(w, j, h) \equiv 0$ otherwise $\operatorname{Test}_{w}^{\prime}(w, j, h) \equiv \overline{t_{w+1}}$. In both cases Test ${ }_{w}^{\prime} \sqsubseteq^{\phi_{h}}$ Test $_{w+1}^{\prime}$. Similarly we can show that TestOT $T_{w}^{\prime} \sqsubseteq^{\phi_{h}} \operatorname{TestOT}_{w+1}^{\prime}$.

Since $\sqsubseteq_{h}^{\phi_{h}}$ is a precongruence, we can replace the Test $O T_{w}^{\prime}$ sub-processes in $I_{w}^{\prime}$ by TestOT $T_{w+1}^{\prime}$, and the Test ${ }_{w}^{\prime}$ sub-processes (except the one for $j=h(w)$ ), by $T e s t_{w+1}^{\prime}$. If $K$ is the resulting process, we have that $I_{w}^{\prime} \sqsubseteq^{\phi_{h}} K$. Let $l=h(w)$. Now $K$ and $I_{w+1}^{\prime}$ differ only in $\operatorname{Test}_{w}^{\prime}(w, l, h)$ process. However Test ${ }_{w}^{\prime}$ is not smaller than Test ${ }_{w+1}^{\prime}$ so we cannot replace the first by the second.

In order to overcome this problem we notice that $r_{w j}^{\prime}$ is the $j$-th element of $r_{w}^{\prime}$ which was received through the $c_{s a_{w}}$ channel. Since we suppose that tests do not contain any of the $c_{s a_{i}}$ channels, $r_{w}^{\prime}$ must have been transfered using the Oblivious Transfer server $S\left(b s_{w}, s a_{w}\right)$. This process receives two values $m_{1}^{\prime}, m_{2}^{\prime}$ and sends one of them, each with probability one half. We suppose that $m_{1}^{\prime}=b_{w}$ and $m_{2}^{\prime}=b_{w+n}$, that is the correct $w$-th path of $B$ has been sent by the Oblivious Transfer (otherwise TestOT $(w)$ would stall with probability at least one half and we could easily prove $\left.I_{w}^{\prime} \sqsubseteq^{\phi_{h}} I_{w+1}^{\prime}\right)$. So $r_{w}^{\prime}$ will be equal to $b_{w}$ (and $k_{w}^{\prime}=1$ ) or $b_{w+n}\left(\right.$ and $\left.k_{w}^{\prime}=2\right)$, each with probability one half. Also $r_{w l}^{\prime}$ will be equal to $b_{w l}$ or $b_{(w+n) l}$. Depending on the outcome of the Oblivious Transfer we will have one of the following cases for Test ${ }_{w}^{\prime}$, where all variables have been instantiated to their values:

$$
\begin{aligned}
& P_{1}=[1 \text { is } 1]\left[b_{w l} \text { is } d_{w l}^{\prime}\right] \overline{t_{w+1}} \star \mid[1 \text { is } 2]\left[b_{w l} \text { is } d_{(w+n) l}^{\prime}\right] \overline{t_{w+1}} \star \\
& P_{2}=[2 \text { is } 1]\left[b_{(w+n) l} \text { is } d_{w l}^{\prime}\right] \overline{t_{w+1}} \star \mid[2 \text { is } 2]\left[b_{(w+n) l} \text { is } d_{(w+n) l}^{\prime}\right] \overline{t_{w+1}}
\end{aligned}
$$

Also let

$$
Q=\operatorname{Test}_{w+1}^{\prime}(w, l, h)=\overline{t_{w+1}} \star+_{0.5}
$$

We show that $P_{1}+{ }_{0.5} P_{2} \sqsubseteq{ }^{\phi_{h}} Q+{ }_{0.5} Q$. Both processes can only perform a $\overline{t_{w+1}} \star$ action so we only have to check the probability of passing $O=t_{w+1}(x)$. By lemma 1 we have $P_{1}+{ }_{0.5} P_{2}\lceil O\rceil=0.5 P_{1}\lceil O\rceil+0.5 P_{2}\lceil O\rceil$. From the 4 couples of tests 2 always fail. Moreover the other two, $\left[b_{w l}\right.$ is $\left.d_{w l}^{\prime}\right]$ and $\left[b_{w l}\right.$ is $\left.d_{(w+n) l}^{\prime}\right]$, cannot both succeed because this would mean that the original test sent both $b_{w h(w)}$ and $b_{(w+n) h(w)}$ and this violates the $\phi_{h}$ condition. Thus one of $P_{1}\lceil O\rceil, P_{2}\lceil O\rceil$ will be 0 so $P_{1}+{ }_{0.5} P_{2}\lceil O\rceil \leq 0.5$. On the other hand $Q+{ }_{0.5} Q\lceil O\rceil=0.5$ so

$$
P_{1}+{ }_{0.5} P_{2} \sqsubseteq^{\phi_{h}} Q+{ }_{0.5} Q
$$

Finally let $C$ be a context constructed from $K$ by replacing $T e s t_{w}^{\prime}$ with a hole (that is $K=C\left[\right.$ Test $\left._{w}^{\prime}\right]$ ). Since $P_{1}, P_{2}$ are the two possible instances of Test ${ }_{w}^{\prime}$, depending on the outcome of the choice performed by the Oblivious Transfer, $K$ will behave similarly to $C\left[P_{1}\right]+{ }_{0.5} C\left[P_{2}\right]$. Moreover $C[Q]+{ }_{0.5} C[Q]$ will behave similarly to $I_{w+1}^{\prime}$ since $I_{w+1}=C\left[\right.$ Test $\left._{w+1}^{\prime}\right]$ and $Q=$ Test $_{w+1}^{\prime}$. By corollary 2 (extended to the $\sqsubseteq^{\phi}$ relation) and equation $(7)$ we have $C\left[P_{1}\right]+{ }_{0.5} C\left[P_{2}\right] \sqsubseteq^{\phi_{h}}$ $C[Q]+{ }_{0.5} C[Q]$ which implies $K \sqsubseteq{ }^{\phi_{h}} I_{w+1}^{\prime}$.

Since $I_{w}^{\prime} \sqsubseteq{ }^{\phi_{h}} K$, the equation (6) is true and we can finish the proof by an induction on $w$. 